\title{
乌ु \\ Theory of electronic transport in random alloys with short-range order: Korringa-Kohn-Rostoker nonlocal coherent potential approximation
}

\author{
P. R. Tulip and J. B. Staunton \\ Department of Physics, University of Warwick, Coventry CV4 7AL, United Kingdom \\ S. Lowitzer, D. Ködderitzsch, and H. Ebert \\ Department Chemie und Biochemie, Physikalische Chemie, Universität München, Butenandtstrasse 5-13, D-81377 München, Germany
}

(Received 14 December 2007; revised manuscript received 6 March 2008; published 11 April 2008)

\begin{abstract}
We present an ab initio formalism for the calculation of transport properties in compositionally disordered systems within the framework of the Korringa-Kohn-Rostoker nonlocal coherent potential approximation. Our formalism is based on the single-particle Kubo-Greenwood linear response and provides a natural means of incorporating the effects of short-range order upon the transport properties. We demonstrate the efficacy of the formalism by examining the effects of short-range order and clustering upon the transport properties of disordered AgPd and CuZn alloys.
\end{abstract}

DOI: 10.1103/PhysRevB.77.165116

PACS number(s): 72.10.-d, 71.20.Be, 72.15.Eb

\section{INTRODUCTION}

The Korringa-Kohn-Rostoker coherent potential approximation $^{1}$ (KKR-CPA) represents an extremely successful one-electron theory capable of describing the properties of many compositionally disordered alloy systems. In particular, in combination with density functional theory ${ }^{2-5}$ (DFT) and the Korringa-Kohn-Rostoker method of band theory, ${ }^{6-8}$ it provides a fully first-principles description of such systems. A long history of successful applications ${ }^{9-14}$ attests to the utility and accuracy to which the method is capable.

Of particular relevance to our discussion here, the KKRCPA has been used to calculate the transport properties of such alloys. Historically, the early work concerning this topic involved the use of a Boltzmann equation, ${ }^{15-17}$ and although these works demonstrated remarkable agreement with experiment, they did suffer from two notable defects: namely, the requirement that well-defined energy bands exist within the alloy, and the neglect of vertex, or "scattering-in," terms. The former defect limits the application of such a theory to weak-scattering alloys only, while the latter could be expected to lead to significant error in systems such as those where appreciable $s-p$ or $s$ - $d$ scattering manifests itself.

Velický ${ }^{18}$ developed a CPA theory for transport using the Kubo-Greenwood ${ }^{19,20}$ formalism as his starting point, which was applied to a two-level tight-binding Hamiltonian. Although capable of yielding vertex corrections, this approach suffered from difficulties when applied to realistic systems. For example, the necessity of assuming that the wave functions are identical on all lattice sites, irrespective of the occupying atomic species. The seminal work of Butler ${ }^{21}$ resolved these issues, as he developed a KKR-CPA theory based on the Kubo-Greenwood linear response formalism. ${ }^{19,20}$ As a multiple-scattering based approach, this did not require the existence of well-defined energy bands, and further, Butler demonstrated how the vertex corrections arose quite naturally within his formalism. Although the formal developments of this work followed Velický's quite closely, the use of a realistic single-electron muffin-tin Hamiltonian allowed connection with first-principles meth- ods to be made. The method has been applied with success to a range of alloy systems $\mathrm{s}^{22,23}$ and it has also been successfully extended to the relativistic regime. ${ }^{24}$

Of course, all of these calculations suffer from the main drawback of the CPA; namely, that as a single-site meanfield theory, it is incapable of incorporating the effects of local-environment fluctuations in the alloy crystal potential. The CPA therefore explicitly ignores the effects of such short-range order (SRO) effects upon the physics of disordered alloys. As discussed by Gonis, ${ }^{25}$ these statistical fluctuations can be important. While in general the presence of SRO is likely to diminish the resistivity, there are examples, the so-called "Komplex" $K$-state alloys, ${ }^{26}$ where the onset of SRO is accompanied by an increase in the alloy residual resistivity.

Recently, there have been some successful attempts at calculating the effects of SRO upon the electronic structure of disordered alloys and they provide the means to study transport properties. Mookerjee and co-workers, ${ }^{27-29}$ using a tight-binding, linear muffin tin orbitals (TB-LMTO) method $^{30}$ in conjunction with an augmented space formalism ${ }^{31,32}$ and real space recursion method, ${ }^{33}$ described SRO effects on alloy electronic densities of states and related quantities, while Saha et al. ${ }^{34}$ obtained spectral functions within the same framework. Recently, Tarafder et al. ${ }^{35}$ developed a formalism for the optical conductivity and reflectivity from the same basis and used it to study copper-zinc alloys. To date, however, it has not been possible to incorporate this technique fully within electronic density functional theory. The recent work of Rowlands et al. ${ }^{36-38}$ along with Biava and co-workers et al. ${ }^{39,40}$ concern a development which is not restricted in this way. References 36 and 37 formulate and illustrate a successful method for incorporating the effects of SRO within the framework of KKR-CPA theory, while implementation for realistic systems is described in Refs. 37 and 39. The method can be readily combined with density functional theory to provide a first-principles description of disordered alloys, as demonstrated in Refs. 38 and 41. This nonlocal CPA (NLCPA) theory is based on reciprocal space coarse-graining ideas introduced by Jarrell and 
Krishnamurthy, ${ }^{42}$ originating from the dynamical cluster approximation (DCA). ${ }^{43-45}$ The KKR-NLCPA ${ }^{36,37}$ introduces an effective (translationally invariant) disorder term $\delta G$, which represents an effective propagator that accounts for all nonlocal scattering correlations on the electronic propagation due to disorder configurations and modifies the structure constants accordingly. By coarse-graining reciprocal space, one naturally introduces real space periodically repeating clusters. As such, the NLCPA maps an effective lattice problem to that of an impurity cluster embedded in a selfconsistently determined effective medium, and thus yields a cluster generalization of the KKR-CPA that includes nonlocal correlations up to the range of the cluster size. Unlike other cluster approaches, such as the molecular CPA ${ }^{46}$ it is fully translationally invariant, that is, the effective medium has the site-to-site translational invariance of the underlying lattice. It is also computationally tractable, largely on account of the reciprocal space coarse-graining procedure employed.

Thus far, the NLCPA has been employed to investigate the effects of SRO upon the electronic structure of a range of realistic systems by using both the muffin-tin Hamiltonian ${ }^{37,47}$ and the tight-binding approach. ${ }^{48}$ Given its ability to successfully address such issues and its proven incorporation into an electronic DFT ${ }^{38}$ for disordered systems with SRO, it makes sense then to extend it to the calculation of transport properties. To this end, by invoking time dependent $\mathrm{DFT}^{49}$ within the adiabatic approximation, in this paper, we present a formalism for the determination of the residual resistivity in the KKR-NLCPA and explicitly demonstrate the efficacy of the method through application to several realistic alloy systems. Our theoretical formalism is a careful generalization of that of Butler ${ }^{21}$ and where appropriate we omit the steps in the derivation which can be straightforwardly obtained from this paper. Our paper is structured as follows: The next section gives a short overview of the conductivity tensor and then the transport coefficients available from the Kubo-Greenwood formalism. This is followed by a section containing the salient points of the KKR-NLCPA formalism including its use in implementing the density functional theory. We then develop our theory for the conductivity of disordered systems with short-range order, which includes the treatment of "vertex corrections." The implementation strategy is outlined before calculations for the effects of SRO on the resistivity of both bcc $\mathrm{CuZn}$ and fcc AgPd are presented.

\section{CONDUCTIVITY: KUBO-GREENWOOD LINEAR RESPONSE}

The Kubo-Greenwood ${ }^{19,20}$ linear response formalism states that, for a disordered system, the symmetric part of the conductivity tensor has coefficients $C$, which can be determined from the evaluation of an expression of the form

$$
C=\operatorname{Tr}\left\langle O_{1} G O_{2} G\right\rangle,
$$

where $G$ is a single-particle Green's function, which is dependent on the details of the effective one-electron potential. $O_{1}$ and $O_{2}$ are operators, and the angled brackets denote a configuration average over the distribution of the potentials.

To determine the dc conductivity, we consider the following expression ${ }^{19-21}$

$$
\sigma_{\mu \nu}\left(E_{F}\right)=\frac{\pi \hbar}{N \Omega}\left\langle\sum_{m n} J_{m n ; \mu} J_{n m ; \nu} \delta\left(E_{F}-E_{m}\right) \delta\left(E_{F}-E_{n}\right)\right\rangle,
$$

where $J_{m n ; \mu}=\left\langle m\left|J_{\mu}\right| n\right\rangle$ denotes the matrix element of the current operator in the $\mu$ th spatial direction, which is given by

$$
J_{\mu}=-i \hbar \frac{e}{m} \frac{\partial}{\partial r_{\mu}},
$$

with $|m\rangle$ and $|n\rangle$ denoting the eigenfunctions of a particular configuration of the disordered system. Here, $N$ is the number of atoms and $\Omega$ is the volume per atom. $E_{F}$ is the Fermi energy.

Within the KKR approach, the electronic structure of the alloy is expressed in terms of the single-particle Green's function, rather than in terms of eigenstates and eigenvalues of the Hamiltonian; we can introduce the Green's function simply by using the identity ${ }^{50}$

$$
-\pi \sum_{n}|n\rangle\langle n| \delta\left(E-E_{n}\right)=\lim _{\eta \rightarrow 0} \operatorname{Im} G(E+i \eta),
$$

while the awkward imaginary part of the Green's function may be removed by writing

$$
-2 i \pi \sum_{n}|n\rangle\langle n| \delta\left(E-E_{n}\right)=\lim _{\eta \rightarrow 0}[G(E+i \eta)-G(E-i \eta)] .
$$

Inserting this into Eq. (2), yields the following for the conductivity

$$
\begin{aligned}
\sigma_{\mu \nu}= & 1 / 4 \lim _{\eta \rightarrow 0}\left[\widetilde{\sigma}_{\mu \nu}\left(E^{+}, E^{+}\right)-\widetilde{\sigma}_{\mu \nu}\left(E^{-}, E^{+}\right)\right. \\
& \left.-\widetilde{\sigma}_{\mu \nu}\left(E^{+}, E^{-}\right)+\widetilde{\sigma}_{\mu \nu}\left(E^{-}, E^{-}\right)\right],
\end{aligned}
$$

where we define the complex energies as

$$
E^{+}=E_{F}+i \eta, \quad E^{-}=E_{F}-i \eta, \quad \eta \rightarrow 0,
$$

and

$$
\widetilde{\sigma}_{\mu \nu}\left(z_{1}, z_{2}\right)=-\frac{\hbar}{\pi N \Omega} \operatorname{Tr}\left\langle J_{\mu} G\left(z_{1}\right) J_{\nu} G\left(z_{2}\right)\right\rangle,
$$

where $z_{1}$ and $z_{2}$ are each either $E^{+}$or $E^{-}$.

For nonoverlapping effective single electron potentials the Hamiltonian takes the form

$$
H=-\frac{\hbar^{2}}{2 m} \nabla^{2}+\sum_{i} v_{\gamma}\left(\mathbf{r}-\mathbf{R}_{i}\right),
$$

where the atomic positions $\mathbf{R}_{i}$ are fixed, and form a regular lattice. The potentials $v_{\gamma}\left(\mathbf{r}_{i}\right)$ vary from site to site $\left(\mathbf{r}_{i}=\mathbf{r}\right.$ $-\mathbf{R}_{i}$ ) and $\gamma$ is a configuration label.

Within the multiple-scattering theory, the single-particle Green's function for a given configuration $\gamma$ can be written as $^{51}$ 


$$
\begin{aligned}
G_{\gamma}\left(E, \mathbf{r}_{i}, \mathbf{r}_{j}^{\prime}\right)= & 2 m / \hbar^{2}\left(\sum_{\Lambda, \Lambda^{\prime}} Z_{\Lambda, \gamma}^{i}\left(E, \mathbf{r}_{i}\right) \tau_{\gamma, \Lambda, \Lambda^{\prime}}^{i j} Z_{\Lambda^{\prime}, \gamma}^{j, \dagger}\left(E, \mathbf{r}_{j}^{\prime}\right)\right. \\
& \left.-\sum_{\Lambda} Z_{\Lambda, \gamma}^{i}\left(\mathbf{r}_{i}^{<}\right) J_{\Lambda, \gamma}^{i \dagger}\left(E, \mathbf{r}_{i}^{>}\right) \delta_{i j}\right)
\end{aligned}
$$

where $\tau_{\gamma, \Lambda, \Lambda}^{i j}$ is the scattering path operator (SPO) describing propagation between sites $i$ and $j$ in configuration $\gamma$, $Z_{\Lambda, \gamma}^{i}\left(E, \mathbf{r}_{i}\right)$ is the regular solution to the Schrödinger and/or Dirac equation in the cell surrounding the atom $i$, and $J_{\Lambda, \gamma}^{i}\left(E, \mathbf{r}_{i}^{\prime}\right)$ represents the irregular solution within the same cell (note that there should be no confusion with the current matrix elements here). $\Lambda$ encapsulates the appropriate angular momentum quantum numbers. ${ }^{21,24}$

In calculating the conductivity, the second term in the Green's function expression [Eq. (10)] is real and may be omitted, when calculated for a real potential at a real energy. ${ }^{21}$ Thus, the conductivity may be written as

$$
\begin{aligned}
\widetilde{\sigma}_{\mu \nu}\left(z_{1}, z_{2}\right)=- & \frac{4 m^{2}}{\pi N \Omega \hbar^{3}} \sum_{i, j} \sum_{\Lambda_{1}, \Lambda_{2}, \Lambda_{3}, \Lambda_{4}} \\
& \times\left\langle J_{\Lambda_{1}, \gamma}^{i, \gamma}, \mu\right. \\
& \left.\times z_{2}, z_{1}\right) \tau_{\gamma, \Lambda_{2} \Lambda_{3}}^{i j}\left(z_{1}\right) J_{\Lambda_{3} \Lambda_{4}, \nu}^{j, \gamma}\left(z_{1}, z_{2}\right) \\
& \left.\left(z_{2}\right)\right\rangle,
\end{aligned}
$$

with

$$
J_{\Lambda \Lambda^{\prime}, \mu}^{i, \gamma}\left(z, z^{\prime}\right)=-\frac{i e \hbar}{m} \int_{\text {cell }_{i}} d \mathbf{r}_{i} Z_{\Lambda, \gamma}^{i}\left(\mathbf{r}_{i}, z\right) \frac{\partial}{\partial r_{\mu}} Z_{\Lambda^{\prime}, \gamma}^{i}\left(\mathbf{r}_{i}, z^{\prime}\right),
$$

where $c e l l$ defines the region surrounding the site $i$.

We now need to consider how to carry out the averaging over configurations implicit in Eq. (11). Butler ${ }^{21}$ showed in detail how to use the CPA to accomplish this. The single site nature of this effective medium theory means, however, that the potentials on the different lattices could only be treated as statistically independent. We will show how to carry out the averaging using the NLCPA whereby short-ranged correlations can be naturally included. To this end, in the next section, we summarize briefly the key aspects we need from the KKR-NLCPA together with its incorporation into electronic density functional theory. Full details can be found in Refs. 36-38 and 41

\section{THE KORRINGA-KOHN-ROSTOKER NONLOCAL COHERENT POTENTIAL APPROXIMATION AND ELECTRONIC DENSITY FUNCTIONAL THEORY}

In general the SPO between two sites $i$ and $j$ for an electron, moving through an effective medium so that it mimics the average motion in a disordered system, is given by

$$
\hat{\tau}^{i j}=\hat{t} \delta_{i j}+\sum_{k \neq i} \hat{t}\left[G\left(\mathbf{R}_{i}-\mathbf{R}_{k}\right)+\delta \hat{G}\left(\mathbf{R}_{i}-\mathbf{R}_{k}\right)\right] \hat{\tau}^{k j} .
$$

Here, all quantities are matrices in angular momentum space and the indices $i$ and $j$ run over all sites in the lattice. $G\left(\mathbf{R}_{i}-\mathbf{R}_{k}\right)$ 's are structure constants. The effective medium is specified by single site $t$-matrices $\hat{t}$ and effective structure constant corrections $\delta \hat{G}\left(\mathbf{R}_{i}-\mathbf{R}_{j}\right)$. The effective medium must be translationally invariant so that $\hat{\tau}^{i j}$ is given in terms of a Brillouin zone integral,

$$
\hat{\tau}^{i j}=\frac{1}{\Omega_{B Z}} \int_{\Omega_{B Z}} d \mathbf{k}\left[\hat{t}^{-1}-G(\mathbf{k})-\delta \hat{G}(\mathbf{k})\right]^{-1} e^{i \mathbf{k} \cdot\left(\mathbf{R}_{i}-\mathbf{R}_{j}\right)} .
$$

In order to establish a tractable procedure for determining the effective medium the NLCPA draws its chief idea from the DCA for interacting electron systems. ${ }^{42,43}$ This is a coarse graining of $\delta \hat{G}$ consistently in real and reciprocal space and a mapping to a self-consistently embedded impurity cluster problem with appropriate boundary conditions imposed. ${ }^{48}$ The full translational symmetry of the underlying lattice is preserved. The size of the cluster sets the range of correlations that can be included. The lattice is divided into "tiles" centered on a superlattice vectors $\mathbf{R}_{C}$ and each contains $N_{c}$ sites at positions $\mathbf{R}_{C}+\mathbf{R}_{I}, I=1, \ldots, N_{c}$. The Brillouin zone is also broken into $N_{c}$ tiles, of volume $\Omega_{t}=\Omega_{B Z} / N_{c}$, centered on the cluster momenta $\mathbf{K}_{n}, n=1, \ldots, N_{c}$ and $\mathbf{R}_{I}$ 's and $\mathbf{K}_{n}$ satisfy the following equation:

$$
\frac{1}{N_{c}} \sum_{\mathbf{K}_{n}} e^{i \mathbf{K}_{n} \cdot\left(\mathbf{R}_{I}-\mathbf{R}_{J}\right)}=\delta_{I J}
$$

Here, $\delta \hat{G}(\mathbf{k})$ is coarse grained so that it has the average value $\delta \hat{G}\left(\mathbf{K}_{n}\right)$ in a tile centered on $\mathbf{K}_{n}$ and in real space, $\delta \hat{G}\left(\mathbf{R}_{I}\right.$ $\left.-\mathbf{R}_{J}\right)=\left(1 / N_{c}\right) \sum_{\mathbf{K}_{n}} \delta \hat{G}\left(\mathbf{K}_{n}\right) e^{i \mathbf{K}_{n} \cdot\left(\mathbf{R}_{I}-\mathbf{R}_{J}\right)} \quad$ with $\delta \hat{G}\left(\mathbf{K}_{n}\right)$ $=\sum_{J \neq I} \delta \hat{G}\left(\mathbf{R}_{I}-\mathbf{R}_{J}\right) e^{-i \mathbf{K}_{n} \cdot\left(\mathbf{R}_{I}-\mathbf{R}_{J}\right)}$.

The SPO is coarse grained,

$$
\hat{\tau}\left(\mathbf{K}_{n}\right)=\frac{N_{c}}{\Omega_{B Z}} \int_{\Omega_{t}} d \tilde{\mathbf{k}}\left[\hat{t}^{-1}-G\left(\tilde{\mathbf{k}}+\mathbf{K}_{n}\right)-\delta \hat{G}\left(\mathbf{K}_{n}\right)\right]^{-1},
$$

appropriate to the reciprocal space tile of volume $\Omega_{t}$ and in real space for multiple scattering starting and ending on cluster sites $I$ and $J$, respectively,

$$
\begin{aligned}
\hat{\tau}^{J J}= & \frac{1}{\Omega_{B Z}} \sum_{\mathbf{K}_{n}}\left(\int _ { \Omega _ { t } } \tilde { d \mathbf { k } } \left[\hat{t}^{-1}-G\left(\tilde{\mathbf{k}}+\mathbf{K}_{n}\right)\right.\right. \\
& \left.\left.-\delta \hat{G}\left(\mathbf{K}_{n}\right)\right]^{-1}\right) e^{i \mathbf{K}_{n} \cdot\left(\mathbf{R}_{I}-\mathbf{R}_{J}\right)} .
\end{aligned}
$$

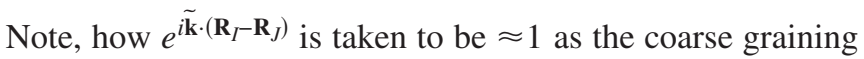
is applied. ${ }^{43}$ The final step is to find the SPO for an impurity cluster $\tau_{\gamma}^{J}$ describing a particular configuration $\gamma_{C}$ of atoms, which is embedded into the NLCPA medium. By demanding that the average is equal to the SPO of the NLCPA enables the effective $t$ matrix and structure constant corrections to be determined, i.e.,

$$
\sum_{\gamma_{C}} P_{\gamma_{C}} \tau_{\gamma_{C}}^{J J}=\hat{\tau}^{I J}
$$

SRO can be included by choosing the probabilities $P_{\gamma_{C}}$ appropriately as demonstrated in, for example, Refs. 37 and 38

In Ref. 38, in a generalization of the work of Johnson et $a l .,{ }^{4,5}$ it is described how to specify a configurationally averaged electronic Grand potential $\bar{\Omega}$ in terms of KKR-NLCPA 
quantities and charge densities $\rho_{\gamma_{C}}\left(\mathbf{r}_{I}\right)$ and one-electron potentials $v_{\gamma_{C}}\left(\mathbf{r}_{I}\right)$ different for each cluster configuration. The functional minimization of $\bar{\Omega}$ with respect to the charge densities, $\rho_{\gamma_{C}}\left(\mathbf{r}_{I}\right)$ 's, determines the total energy of the system and requires $\rho_{\gamma_{C}}\left(\mathbf{r}_{I}\right)$ 's and $v_{\gamma_{C}}\left(\mathbf{r}_{I}\right)$ 's to be found self-consistently. Rowlands et al. ${ }^{38}$ applied this DFT to investigate how the total energy, charge densities, and densities of states are affected by SRO. Tulip et al. ${ }^{47}$ showed how further information can be found about the effects of SRO on the electronic structure by formulating and calculating the Bloch spectral function at the cluster momenta and averaged over tiles, while Batt and Rowlands ${ }^{48}$ explained how the spectral function at any point in the Brillouin zone can be found. In the following, we build on these developments and describe the theory for a two-particle correlation function of a disordered system with SRO. The particular example is to the dc conductivity.

\section{ANALYTIC CONFIGURATION AVERAGING OF THE CONDUCTIVITY USING THE KORRINGA-KOHN- ROSTOKER NONLOCAL COHERENT POTENTIAL APPROXIMATION}

From the above, it is clear that the KKR-NLCPA should enable an analytical configurational average of the conduc-

tivity to be carried out. To do so, some care needs to be exercised. There are two distinct cases that we must consider in Eq. (11): (i) where the two sites under consideration, $i$ and $j$, lie within the same NLCPA cluster, and (ii) when they lie in two different clusters, in which case the occupancies of the sites will be statistically independent, as the two distinct clusters will be statistically independent. This is a natural generalization of Butler's work, ${ }^{21}$ where he distinguishes between the two cases of $i=j$ and $i \neq j$.

Hereon, we use lower case letters to denote general sites in the lattice, $i, j, \ldots$, upper case $C$ denotes tiles containing the clusters, and upper case letters, with the exception of $C$, denote sites within clusters. So for a site at position $\mathbf{R}_{i}$, we use $\mathbf{R}_{i}=\mathbf{R}_{C}+\mathbf{R}_{I}$ and $I, J, \ldots$ label sites within tile $C, I^{\prime}, J^{\prime}, \ldots$ sites within tile $C^{\prime}$, etc.

We accordingly write

$$
\widetilde{\sigma}_{\mu \nu}\left(z_{1}, z_{2}\right)=\widetilde{\sigma}_{\mu \nu}^{0}\left(z_{1}, z_{2}\right)+\widetilde{\sigma}_{\mu \nu}^{1}\left(z_{1}, z_{2}\right)
$$

$$
\widetilde{\sigma}_{\mu \nu}^{0}\left(z_{1}, z_{2}\right)=-\frac{4 m^{2}}{\pi \hbar^{3} \Omega} \sum_{J \in C} \sum_{\Lambda_{1}, \Lambda_{2}, \Lambda_{3}, \Lambda_{4}}\left\langle J_{\Lambda_{1} \Lambda_{2} ; \mu}^{I}\left(z_{2}, z_{1}\right) \tau_{\Lambda_{2} \Lambda_{3}}^{I J}\left(z_{1}\right) J_{\Lambda_{3} \Lambda_{4} ; \nu}^{J}\left(z_{1}, z_{2}\right) \tau_{\Lambda_{4} \Lambda_{1}}^{J I}\left(z_{2}\right)\right\rangle,
$$

and

$$
\widetilde{\sigma}_{\mu \nu}^{1}\left(z_{1}, z_{2}\right)=-\frac{4 m^{2}}{\pi \hbar^{3} \Omega} \sum_{C^{\prime} \neq C} \sum_{J^{\prime} \in C^{\prime}} \sum_{\Lambda_{1}, \Lambda_{2}, \Lambda_{3}, \Lambda_{4}}\left\langle J_{\Lambda_{1} \Lambda_{2} ; \mu}^{I}\left(z_{2}, z_{1}\right) \tau_{\Lambda_{2} \Lambda_{3}}^{I, C^{\prime}+J^{\prime}}\left(z_{1}\right) J_{\Lambda_{3} \Lambda_{4} ; \nu}^{J^{\prime}}\left(z_{1}, z_{2}\right) \tau_{\Lambda_{4} \Lambda_{1}}^{C^{\prime}+J^{\prime}, I}\left(z_{2}\right)\right\rangle
$$

where $\widetilde{\sigma}^{0}$ includes sites $J$ within the same NLCPA cluster (denoted $C$ ) as our reference site $I$ and $\widetilde{\sigma}^{1}$ includes all sites lying outside this cluster. Note that in writing these equations, we have utilized the translational invariance of the averaged system to remove the second sum appearing in Eq. (11).

We now introduce response functions, such that we can write

$$
\begin{aligned}
& \widetilde{\sigma}_{\mu \nu}^{0}\left(z_{1}, z_{2}\right) \\
& =-\frac{4 m^{2}}{\pi \hbar^{3} \Omega} \sum_{\gamma_{C}} P_{\gamma_{C}} \sum_{\Lambda_{1}, \Lambda_{2}} J_{\Lambda_{1} \Lambda_{2} ; \mu}^{I, \gamma_{C}}\left(z_{2}, z_{1}\right) K_{\Lambda_{2}, \Lambda_{1} ; \nu}^{I ; C, \gamma_{C}}\left(z_{1}, z_{2}\right) \\
& K_{\Lambda_{2}, \Lambda_{1} ; \nu}^{I ; C, \gamma_{C}}\left(z_{1}, z_{2}\right) \\
& \quad=\sum_{J \in C} \sum_{\Lambda_{3}, \Lambda_{4}}\left\langle\tau_{\Lambda_{2} \Lambda_{3}}^{I J}\left(z_{1}\right) J_{\Lambda_{3} \Lambda_{4}}^{J, \gamma_{C}, \nu}\left(z_{1}, z_{2}\right) \tau_{\Lambda_{4} \Lambda_{1}}^{J I}\left(z_{2}\right)\right\rangle_{C, \gamma_{C}}
\end{aligned}
$$

Here, $K_{\Lambda_{2}, \Lambda_{1} ; \nu}^{I ; C, \gamma_{C}}\left(z_{1}, z_{2}\right)$ involves an average over all configura- tions, except the configuration is fixed in cluster $C$ to be $\gamma_{C}$. The single site quantity $\left.J_{\Lambda_{1} \Lambda_{2} ; \mu}^{I, \gamma_{C}}, z_{2}, z_{1}\right)$ is set up depending on what kind of element occupies site $I$ and the one-electron potential $v_{\gamma_{C}}\left(\mathbf{r}_{I}\right)$ that is dependent on the configuration $\gamma_{C}$.

Similarly, for the intercluster contributions to the conductivity, we can introduce the following:

$$
\begin{aligned}
\tilde{\sigma}_{\mu \nu}^{1}\left(z_{1}, z_{2}\right)=- & \frac{4 m^{2}}{\pi \hbar^{3} \Omega} \sum_{C^{\prime} \neq C} \sum_{\gamma_{C}} P_{\gamma_{C}} \sum_{\gamma_{C^{\prime}}} P_{\gamma_{C^{\prime}}} \\
& \times \sum_{\Lambda_{1}, \Lambda_{2}} J_{\Lambda_{1} \Lambda_{2} ; \mu}^{I, \gamma_{C}}\left(z_{2}, z_{1}\right) L_{\Lambda_{2}, \Lambda_{1} ; \nu}^{I, C, \gamma_{C} ; C^{\prime}, \gamma_{C^{\prime}}\left(z_{1}, z_{2}\right),}
\end{aligned}
$$

and

$$
\begin{aligned}
L_{\Lambda_{2}, \Lambda_{1} ; \nu}^{I, C, \gamma_{C} ; C^{\prime}, \gamma_{C^{\prime}}}\left(z_{1}, z_{2}\right)= & \sum_{J^{\prime} \in C^{\prime}} \sum_{\Lambda_{3}, \Lambda_{4}}\left\langle\tau_{\Lambda_{2} \Lambda_{3}}^{I, C^{\prime}+J^{\prime}}\left(z_{1}\right) J_{\Lambda_{3} \Lambda_{4} ; \nu}^{J^{\prime}, \gamma_{C^{\prime}}}\left(z_{1}, z_{2}\right)\right. \\
& \left.\times \tau_{\Lambda_{4} \Lambda_{1}}^{C^{\prime}+J^{\prime}, I}\left(z_{2}\right)\right\rangle_{C, \gamma_{C} ; C^{\prime}, \gamma_{C^{\prime}}}
\end{aligned}
$$

where the notation is similar to before but the average now 
fixes cluster $C$ to be occupied by configuration $\gamma_{C}$ and cluster $C^{\prime}$ to be loaded with configuration $\gamma_{C^{\prime}}$.

In order to evaluate the ensemble averages contained in Eqs. (22)-(25), it is helpful to express the SPO $\tau$ for a particular configuration as the SPO in the NLCPA medium plus corrections. Again, this is the direct generalization of Butler's approach. ${ }^{21}$ We can write

$$
\tau^{i j}=\hat{\tau}^{i j}+\sum_{k, l} \hat{\tau}^{i k} T^{k l} \hat{\tau}^{j j}
$$

where the effective medium path SPO is denoted $\hat{\tau}$ as before and $T$ is the total scattering matrix relevant to the specific configuration. The double summation is taken over all lattice sites. $\tau^{i j}$ satisfies the following equation:

$$
\sum_{k}\left[t_{k}^{-1} \delta_{i k}-G\left(\mathbf{R}_{i}-\mathbf{R}_{k}\right)\right] \tau^{k j}=\delta_{i j},
$$

where all quantities are matrices in angular momentum space. We consider fluctuations about the NLCPA medium to obtain

$$
\begin{aligned}
\sum_{k}[ & {\left[\left(t_{k}^{-1}-\hat{t}^{-1}\right) \delta_{i k}+\delta \hat{G}\left(\mathbf{R}_{i}-\mathbf{R}_{k}\right)+\hat{t}^{-1}\right.} \\
& \left.-\delta \hat{G}\left(\mathbf{R}_{i}-\mathbf{R}_{k}\right)-G\left(\mathbf{R}_{i}-\mathbf{R}_{k}\right)\right] \tau^{k j}=\delta_{i j},
\end{aligned}
$$

which can be rearranged to yield

$$
\tau^{i j}=\hat{\tau}^{i j}-\sum_{k, l} \hat{\tau}^{i k} \Delta m^{k l} \tau^{l j} .
$$

with

$$
\Delta m^{k l}=\left(t_{k}^{-1}-\hat{t}^{-1}\right) \delta_{k l}-\delta \hat{G}\left(\mathbf{R}_{k}-\mathbf{R}_{l}\right)
$$

and have used the fact that the effective medium SPO may be written as the inverse of the matrix with elements $\left[\hat{t}^{-1} \delta_{i j}\right.$ $\left.-\delta \hat{G}\left(\mathbf{R}_{i}-\mathbf{R}_{j}\right)-G\left(\mathbf{R}_{i}-\mathbf{R}_{j}\right)\right]$.

We can thus write down

$$
\sum_{l} T^{k l} \hat{\tau}^{j j}=\sum_{l} \Delta m^{k l} \tau^{l j}
$$

If we now substitute for $\tau^{j j}$ using Eq. (26), label the sites according to clusters, $C$ and sites within those clusters (uppercase letters), we obtain

$$
T^{C+K, C^{\prime}+L^{\prime}}=x^{K L} \delta_{C, C^{\prime}}+\sum_{C^{\prime \prime} \neq C} \sum_{M N^{\prime \prime}} x^{K M} \hat{\tau}^{C+M, C^{\prime \prime}+N^{\prime \prime}} T^{C^{\prime \prime}+N^{\prime \prime}, C^{\prime}+L^{\prime}},
$$

where we have introduced the matrix $x$ associated with a single cluster of sites given by

$$
x^{I J}=\sum_{K}(1+\Delta m \hat{\tau})_{I K}^{-1} \Delta m^{K J} .
$$

Our results here are a direct cluster generalization of Butler. ${ }^{21}$ Note also that these results are consistent with the cluster CPA conductivity formalism of Hwang et al. ${ }^{52}$ (although that is phrased in terms of $t$-matrices, rather than the $x$ matrix that we use in this work). Further, the special case of a single-site cluster recovers the more familiar CPA results.

The NLCPA amounts to writing

$$
\begin{gathered}
\left\langle T^{C+K, C^{\prime}+L^{\prime}}\right\rangle_{N L C P A}=\left\langle x^{K L}\right\rangle \delta_{C, C^{\prime}}+\sum_{C^{\prime \prime} \neq C} \sum_{M N^{\prime \prime}}\left\langle x^{K M}\right\rangle \hat{\tau}^{C+M, C^{\prime \prime}+N^{\prime \prime}} \\
\times\left\langle T^{C^{\prime \prime}+N^{\prime \prime}, C^{\prime}+L^{\prime}}\right\rangle_{N L C P A}
\end{gathered}
$$

and if we choose that $\left\langle x^{I J}\right\rangle=0$, which is another way of expressing the NLCPA ansatz [Eq. (18)], then $\langle T\rangle_{N L C P A}=0$, and we obtain $\langle\tau\rangle_{N L C P A}=\hat{\tau}$ [Eq. (18)]. Of course, in writing this, we have made the approximation that

$$
\begin{aligned}
& \left\langle x^{K M} \hat{\tau}^{C+M, C^{\prime \prime}+N^{\prime \prime}} T^{\left.C^{\prime \prime}+N^{\prime \prime}, C^{\prime}+L^{\prime}\right\rangle}\right. \\
& \quad \approx\left\langle x^{K M}\right\rangle \hat{\tau}^{C+M, C^{\prime \prime}+N^{\prime \prime}}\left\langle T^{C^{\prime \prime}+N^{\prime \prime}, C^{\prime}+L^{\prime}}\right\rangle,
\end{aligned}
$$

which is analogous to the usual CPA-type averaging approximation.

Using Eq. (26) and closely following a cluster generalization of Butler's derivation, which refers to fluctuations about the single site CPA medium, we find (suppressing angular momentum labeling)

$$
K_{\nu}^{I ; C, \gamma_{C}}=\sum_{M, N} D_{I M}^{\gamma_{C}} \tilde{K}_{M N ; \nu}^{C, \gamma_{C}} D_{N I}^{\dagger \gamma_{C}},
$$

with

$$
\widetilde{K}_{I J ; \nu}^{C, \gamma_{C}}=\sum_{K L} \hat{\tau}^{K} \widetilde{J}_{K L ; \nu}^{\gamma_{C}} \hat{\tau}^{J J}+\sum_{C^{\prime \prime} \neq C} \sum_{K^{\prime \prime}, L^{\prime \prime}} \hat{\tau}^{I, C^{\prime \prime}+K^{\prime \prime}} \Gamma_{C^{\prime \prime} ; K^{\prime \prime} L^{\prime \prime} ; \nu}^{C, \gamma_{C}} \hat{\tau}^{C^{\prime \prime}+L^{\prime \prime}, J},
$$

and

$$
L_{\nu}^{I, C, \gamma_{C} ; C^{\prime} \gamma_{C^{\prime}}}=\sum_{M, N} D_{I M}^{\gamma_{C}} \widetilde{L}_{M N ; \nu}^{C, \gamma_{C} ; C^{\prime}, \gamma_{C^{\prime}}} D_{N I}^{\dagger \gamma_{C}},
$$

where

$$
\begin{aligned}
\widetilde{L}_{M N ; \nu}^{C, \gamma_{C} ; C^{\prime}, \gamma_{C^{\prime}}}= & \sum_{K^{\prime}, L^{\prime}} \hat{\tau}^{M, C^{\prime}+K^{\prime}} \widetilde{J}_{K^{\prime} L^{\prime} ; \nu}^{\gamma_{C^{\prime}}} \hat{\tau}^{C^{\prime}+L^{\prime}} \\
& +\sum_{C^{\prime \prime} \neq\left(C, C^{\prime}\right)} \sum_{K^{\prime \prime}, L^{\prime \prime}}\left(\hat{\tau}^{M, C^{\prime \prime}+K^{\prime \prime}} \Gamma_{C^{\prime \prime}, K^{\prime \prime}, L^{\prime \prime} ; \nu}^{C, \gamma_{C} ; C^{\prime}, \gamma_{C^{\prime}}} \hat{\tau}^{C^{\prime \prime}+L^{\prime \prime}, N}\right) .
\end{aligned}
$$

In Eqs. (36) and (38), we use the NLCPA projector $D\left(D^{\dagger}\right)$ which is $D=(1+\Delta m \hat{\tau})^{-1}[(1+\hat{\tau} \Delta m)]$ found in Eqs. (30) and (33). The NLCPA ansatz can be rewritten in terms of them, i.e., $\Sigma_{\gamma_{C}} P_{\gamma_{C}} D^{\gamma_{C}}=1$. We have also defined the current quantities

$$
\widetilde{J}_{K L ; \nu}^{\gamma_{C}}=\sum_{N} D_{K N}^{\dagger \gamma_{C}} J_{\nu}^{N, \gamma_{C}} D_{N L}^{\gamma_{C}}
$$

in Eqs. (37) and (39). Finally, we have introduced vertex functions $\Gamma_{C^{\prime \prime}, K^{\prime \prime}, L^{\prime \prime} ; \nu}^{C, \gamma_{C}}$ and $\Gamma_{C^{\prime \prime}, K^{\prime \prime}, L^{\prime \prime} ; \nu}^{C, \gamma_{C}}$ which are the NLCPA analogs of the vertex functions derived by Butler. ${ }^{21}$ We now show how to calculate these quantities. 


\section{VERTEX FUNCTIONS AND THE CONDUCTIVITY}

Our two vertex functions are slightly different. $\Gamma_{C^{\prime \prime} ; K^{\prime \prime}, L^{\prime \prime} ; \nu}^{C, \gamma_{C}}$ which appears in Eq. (37) for the intracluster component of the conductivity, concerns the connection between the configurational occupation in one cluster $C$ with that of another $C^{\prime \prime}$, whereas the vertex function $\Gamma_{C^{\prime \prime}, K^{\prime \prime}, L^{\prime \prime} ; \nu}^{C, \gamma_{C} ; C^{\prime}, \gamma_{C^{\prime}}}$ for the intercluster contribution relates the contents of cluster $C^{\prime \prime}$ with that in two others, the reference one $C$ and another $C^{\prime}$. To facilitate the derivation of a closed set of equations, we introduce an approximation, analogous to that in Ref. 21, and assume that the dependence of the latter on the contents of cluster $C$ may be neglected. Thus,

$$
\Gamma_{C^{\prime \prime}, K^{\prime \prime}, L^{\prime \prime} ; \nu}^{C, \gamma_{C} ; C^{\prime}, \gamma_{C^{\prime}}}=\Gamma_{C^{\prime \prime}, K^{\prime \prime}, L^{\prime \prime} ; v^{\prime}}^{C^{\prime}, \gamma_{C^{\prime}}}
$$

leading to $\widetilde{L}_{M N ; \nu}^{C, \gamma_{C} ; C^{\prime}, \gamma_{C^{\prime}}}=\widetilde{L}_{M N ; \nu}^{C^{\prime}, \gamma_{C^{\prime}}}$ in Eq. (39). Using Eq. (26), the NLCPA condition [Eq. (18)] (or its equivalent renditions) and tracking the steps in Ref. 21, we obtain

$$
\begin{aligned}
\Gamma_{C, I J ; \nu}^{C^{\prime}, \gamma_{C^{\prime}}=} & \sum_{K, L, M^{\prime}, N^{\prime}}\left\langle x^{I K, \gamma_{C}} \hat{\tau}^{K, C^{\prime}+M^{\prime}} \tilde{J}_{M^{\prime} N^{\prime} ; \nu}^{\gamma_{C^{\prime}}} \hat{\tau}^{C^{\prime}+N^{\prime}, L} x^{L J, \gamma_{C}}\right\rangle_{C^{\prime}, \gamma_{C^{\prime}}} \\
+ & \sum_{C^{\prime \prime} \neq\left(C, C^{\prime}\right)} \sum_{K, L, M^{\prime \prime}, N^{\prime \prime}}\left\langle x^{I K, \gamma_{C} \hat{\tau}^{K, C^{\prime \prime}+M^{\prime \prime}} \Gamma_{C^{\prime \prime}, M^{\prime \prime} N^{\prime \prime} ; \nu}^{C^{\prime}, \gamma^{\prime}}}\right. \\
& \times \hat{\tau}^{C^{\prime \prime}+N^{\prime \prime}, L} x^{\left.L J, \gamma_{C}\right\rangle_{C^{\prime}, \gamma_{C^{\prime}}},}
\end{aligned}
$$

which, if we compare to Eq. (39), allows us to write the vertex function in terms of the response function

$$
\Gamma_{C, I J ; \nu}^{C^{\prime}, \gamma_{C^{\prime}}}=\sum_{K, L}\left\langle x^{I K, \gamma_{C}} \widetilde{L}_{K L ; \nu}^{C^{\prime}, \gamma_{C^{\prime}}} x^{L J, \gamma_{C}}\right\rangle_{C^{\prime}, \gamma_{C^{\prime}}} .
$$

This yields a closed set of equations for the conductivity. We may now write Eqs. (22) and (24) as

$$
\begin{aligned}
\widetilde{\sigma}_{\mu \nu}^{0}\left(z_{1}, z_{2}\right)=- & \frac{4 m^{2}}{\pi \hbar^{3} \Omega N_{c}} \sum_{\gamma_{C}} P_{\gamma_{C}} \\
& \times \sum_{\Lambda_{1}, \Lambda_{2}} \sum_{I, J} \widetilde{J}_{J I, \Lambda_{2}, \Lambda_{1} ; \mu}^{\gamma_{C}}\left(z_{2}, z_{1}\right) \widetilde{K}_{I J, \Lambda_{1}, \Lambda_{2} ; \nu}^{C, \gamma_{C}}\left(z_{1}, z_{2}\right),
\end{aligned}
$$

and

$$
\begin{aligned}
\widetilde{\sigma}_{\mu \nu}^{1}\left(z_{1}, z_{2}\right)=- & \frac{4 m^{2}}{\pi \hbar^{3} \Omega N_{c}} \sum_{C \in C} \sum_{C^{\prime} \neq C} \sum \sum_{J^{\prime} \in C^{\prime}} P_{\gamma_{C}, \gamma_{C^{\prime}}} P_{\gamma_{C}} P_{\gamma_{C^{\prime}}} \\
& \times \sum_{\Lambda_{1}, \Lambda_{2}} \sum_{I, J} \widetilde{J}_{J I, \Lambda_{2}, \Lambda_{1} ; \mu}^{\gamma_{C}}\left(z_{2}, z_{1}\right) \widetilde{L}_{I J, \Lambda_{1}, \Lambda_{2} ; \nu}^{C^{\prime}, \gamma_{C^{\prime}}}\left(z_{1}, z_{2}\right) .
\end{aligned}
$$

The response functions that determine the conductivity are given by

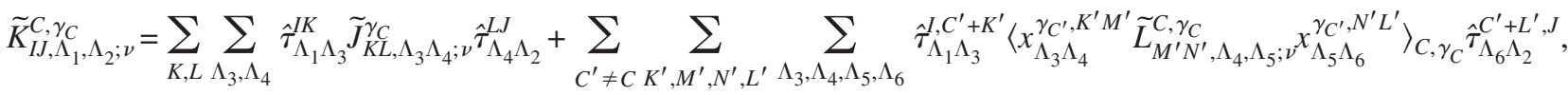

$$
\begin{aligned}
& \tilde{L}_{I J, \Lambda_{1}, \Lambda_{2} ; \nu}^{C^{\prime}, \gamma_{C^{\prime}}}=\sum_{K^{\prime}, L^{\prime}} \sum_{\Lambda_{3}, \Lambda_{4}} \hat{\tau}_{\Lambda_{1} \Lambda_{3} \Lambda^{\prime}+K^{\prime}}^{\prime} \tilde{J}_{K^{\prime} L^{\prime}, \Lambda_{3}, \Lambda_{4} ; \nu}^{\gamma_{C^{\prime}}} \hat{\tau}_{\Lambda_{4} \Lambda_{2}^{\prime}+L_{2}^{\prime}, J} \\
& +\sum_{C^{\prime \prime} \neq\left(C, C^{\prime}\right) K^{\prime \prime}, L^{\prime \prime}, M^{\prime \prime}, N^{\prime \prime}} \sum_{\Lambda_{3}, \Lambda_{4}, \Lambda_{5}, \Lambda_{6}} \hat{\tau}_{\Lambda_{1} C_{3}^{\prime \prime}+K^{\prime \prime}}^{\Lambda_{3}}\left\langle x_{\Lambda_{3} \Lambda_{4}}^{\gamma_{C^{\prime \prime}}, K^{\prime \prime} M^{\prime \prime}} \tilde{L}_{M^{\prime \prime}, N^{\prime \prime}, \Lambda_{4}, \Lambda_{5} ; \nu}^{C^{\prime}, \gamma_{C^{\prime}}} x_{\Lambda_{5} \Lambda_{6}}^{\gamma_{C^{\prime \prime}, N^{\prime \prime}, L^{\prime \prime}}}\right\rangle_{C^{\prime}, \gamma_{C^{\prime}}} \hat{\tau}_{\Lambda_{6} C_{2}^{\prime \prime}+L^{\prime \prime}, J}
\end{aligned}
$$

\section{SOLUTION OF TRANSPORT EQUATION}

The conductivity is determined by solution of the transport equation [Eq. (47)]. We first see that in the key Eqs. (44) and (45), we require the response function $\widetilde{L}_{I J ; \nu}^{C^{\prime}, \gamma_{C^{\prime}}}$ averaged over configurations $\gamma_{C^{\prime}}$ that can be assigned to a cluster $C^{\prime}$ in a NLCPA tile located at position $\mathbf{R}_{C^{\prime}}$ and also that we require this to be summed over all clusters $C^{\prime}$. We rewrite Eq. (47), omitting the quantum numbers, $\Lambda_{1}, \Lambda_{2}$, etc., for brevity, and denote the averages $\Sigma_{\gamma_{C^{\prime}}} P_{\gamma_{C^{\prime}}} \widetilde{L}_{I J ; \nu}^{C^{\prime}, \gamma_{C^{\prime}}}$ and $\sum_{\gamma_{C^{\prime}}} P_{\gamma_{C^{\prime}}} \widetilde{J}_{K^{\prime}, L^{\prime} ; \nu}^{\gamma_{C^{\prime}}}$ as $L_{I J ; \nu}^{C^{\prime}}$ and $J_{K^{\prime}, L^{\prime} ; \nu}$, respectively, as well as summing over clusters $C^{\prime}$,

$$
\begin{aligned}
\sum_{C^{\prime}} L_{I J ; \nu}^{C^{\prime}}= & \sum_{C^{\prime}} \sum_{K^{\prime}, L^{\prime}} \hat{\tau}^{I, C^{\prime}+K^{\prime}} J_{K^{\prime}, L^{\prime} ; \nu} \hat{\tau}^{C^{\prime}+L^{\prime}, J} \\
& -\sum_{K^{\prime}, L^{\prime}} \hat{\tau}^{I, K^{\prime}} J_{K^{\prime}, L^{\prime} ; \nu} \hat{\tau}^{L^{\prime}, J} \\
& +\sum_{C^{\prime}} \sum_{C^{\prime \prime} \neq C^{\prime} K^{\prime \prime}, L^{\prime \prime}, M^{\prime \prime}, N^{\prime \prime}} \hat{\tau}^{, C^{\prime \prime}+K^{\prime \prime}} w^{K^{\prime \prime}, L^{\prime \prime}, M^{\prime \prime}, N^{\prime \prime}} \\
& \times \hat{\tau}^{C^{\prime \prime}+L^{\prime \prime}, J} L_{C^{\prime \prime}, M^{\prime \prime}, N^{\prime \prime} ; \nu}^{C^{\prime}}
\end{aligned}
$$

where $w^{K^{\prime \prime}, L^{\prime \prime}, M^{\prime \prime}, N^{\prime \prime}}=\left\langle x^{K^{\prime \prime}, L^{\prime \prime}} x^{M^{\prime \prime}, N^{\prime \prime}}\right\rangle$ and $L_{I J ; \nu}^{C}$ is defined as zero.

We now write the SPOs in terms of their lattice Fourier transforms, i.e., 


$$
\hat{\tau}^{J, C^{\prime}+J^{\prime}}=\frac{1}{\Omega_{B Z}} \sum_{\mathbf{K}_{n}} \int_{\Omega_{t}} d \tilde{\mathbf{k}} \hat{\tau}\left(\mathbf{K}_{n} ; \tilde{\mathbf{k}}\right) e^{i\left[\left(\mathbf{K}_{n}+\tilde{\mathbf{k}}\right) \cdot\left(\mathbf{R}_{I^{-}} \mathbf{R}_{C^{\prime}}-\mathbf{R}_{J^{\prime}}\right)\right]},
$$

where

$$
\hat{\tau}\left(\mathbf{K}_{n} ; \widetilde{\mathbf{k}}\right)=\left[\hat{t}^{-1}-G\left(\widetilde{\mathbf{k}}+\mathbf{K}_{n}\right)-\delta \hat{G}\left(\mathbf{K}_{n}\right)\right]^{-1}
$$

We also write the response functions $L_{I J ; \nu}^{C^{\prime}}$ in terms of cluster lattice Fourier transforms, i.e.,

$$
L_{I J ; \nu}^{C^{\prime}}=\frac{N_{c}}{\Omega_{B Z}} \int_{\Omega_{t}} d \tilde{\mathbf{k}} L_{I J ; \nu}(\tilde{\mathbf{k}}) e^{i \mathbf{k} \cdot\left(\mathbf{R}_{\left.C^{-}-\mathbf{R}_{C^{\prime}}\right)} .\right.}
$$

On carrying out the sums over $C$ and $C^{\prime}$ in Eq. (48), we thus obtain

$$
\begin{aligned}
L_{I J ; \nu}(0)= & \sum_{K L}\left[\frac{1}{\Omega_{B Z}} \sum_{\mathbf{K}_{n}, \mathbf{K}_{n^{\prime}}} \int_{\Omega_{t}} \tilde{d} \hat{\mathbf{\tau}}\left(\mathbf{K}_{n} ; \tilde{\mathbf{k}}\right) e^{i\left(\mathbf{K}_{n}+\tilde{\mathbf{k}}\right) \cdot\left(\mathbf{R}_{I}-\mathbf{R}_{K}\right)} \hat{\tau}\left(\mathbf{K}_{n^{\prime}} ; \tilde{\mathbf{k}}\right) e^{i\left(\mathbf{K}_{n^{\prime}}+\tilde{\mathbf{k}}\right) \cdot\left(\mathbf{R}_{L^{-}}-\mathbf{R}_{J}\right)}-\hat{\tau}^{I K} \hat{\tau}^{L J}\right] J_{K L ; \nu} \\
& +\sum_{K, L, M, N} w^{K, L, M, N}\left[\frac{1}{\Omega_{B Z}} \sum_{\mathbf{K}_{n}, \mathbf{K}_{n^{\prime}}} \int_{\Omega_{t}} \tilde{\mathbf{k}} \hat{\tau}\left(\mathbf{K}_{n} ; \tilde{\mathbf{k}}\right) e^{i\left(\mathbf{K}_{n}+\tilde{\mathbf{k}}\right) \cdot\left(\mathbf{R}_{I}-\mathbf{R}_{K}\right)} \hat{\tau}\left(\mathbf{K}_{n^{\prime}} ; \tilde{\mathbf{k}}\right) e^{i\left(\mathbf{K}_{n^{\prime}}+\tilde{\mathbf{k}}\right) \cdot\left(\mathbf{R}_{L^{-}}-\mathbf{R}_{J}\right)}-\hat{\tau}^{I K} \hat{\tau}^{L J}\right] L_{M N ; \nu}(0) .
\end{aligned}
$$

Since $L_{I J ; \nu}(0)$ is translationally invariant, $L_{I J, \nu}(0)$ $=L_{I+I_{1}+C_{1}, J+I_{1}+C_{1} ; \nu}(0)$ for a translation by an arbitrary lattice vector $\mathbf{R}_{i}=\mathbf{R}_{C_{1}}+\mathbf{R}_{I_{1}}$ and we can sum it over the $N_{c}$ tile lattice vectors, $L_{I J \nu}(0)=\frac{1}{N_{c}} \sum_{I_{1}} L_{I+I_{1}, J+I_{1} ; \nu}$. Using this manipulation and applying the NLCPA coarse graining again so that $e^{i \tilde{\mathbf{k}} \cdot \mathbf{R}_{I}} \approx 1$, we find

$$
L_{I J ; \nu}(0)=\sum_{K, L} \chi^{I, K, L, J} J_{K L ; \nu}+\sum_{K, L, M, N} w^{K, L, M, N} \chi^{I, K, L, J} L_{M N ; \nu}(0),
$$

with the $\chi$ involving a convolution integral over the Brillouin zone,

$$
\begin{aligned}
\chi^{I, K, L, J}= & \frac{1}{\Omega_{B Z}} \sum_{\mathbf{K}_{n}} \int_{\Omega_{t}} d \tilde{\mathbf{k}} \hat{\tau}\left(\mathbf{K}_{n} ; \tilde{\mathbf{k}}\right) \\
& \times e^{i \mathbf{K}_{n} \cdot\left(\mathbf{R}_{I}-\mathbf{R}_{K}\right)} \hat{\tau}\left(\mathbf{K}_{n} ; \tilde{\mathbf{k}}\right) e^{i \mathbf{K}_{n} \cdot\left(\mathbf{R}_{L}-\mathbf{R}_{J}\right)}-\hat{\tau}^{K} \hat{\tau}^{L J} .
\end{aligned}
$$

Here, $L_{I J}(0)$ is extracted by inverting a super matrix [1 $-w \chi]$, which has a dimension $N_{c} \times N_{c} \times N_{\Lambda} \times N_{\Lambda}$ ( $N_{\Lambda}$ specifying the number of angular momentum quantum numbers).

By comparing Eqs. (46) and (47) and using the definition $L_{I J ; \nu}^{C}=0$, the second term on the right hand side of Eq. (46) vanishes. We thus find the intercluster contribution to the conductivity to be

$$
\begin{aligned}
\tilde{\sigma}_{\mu \nu}^{1}= & -\frac{4 m^{2}}{\pi \hbar^{3} \Omega} \sum_{I, J, K, L} \sum_{\Lambda_{1}, \Lambda_{2}, \Lambda_{3}, \Lambda_{4}} J_{L I, \Lambda_{1}, \Lambda_{2} ; \mu}([1 \\
& \left.-\chi(\mathbf{0}) w]^{-1} \chi(\mathbf{0})\right)_{\Lambda_{1}, \Lambda_{3}, \Lambda_{4}, \Lambda_{2}}^{J K L} J_{J K, \Lambda_{3}, \Lambda_{4} ; \nu},
\end{aligned}
$$

and the intracluster component to be

$$
\begin{aligned}
\widetilde{\sigma}_{\mu \nu}^{0}= & \frac{4 m^{2}}{\pi \hbar^{3} \Omega} \sum_{\gamma_{C}} P_{\gamma_{C}} \sum_{I, K, L} \\
& \times \sum_{\Lambda_{1}, \Lambda_{2}, \Lambda_{3}, \Lambda_{4}} J_{I, \Lambda_{4}, \Lambda_{1} ; \mu}^{\gamma_{C} ; \hat{\tau}_{\Lambda_{1}, \Lambda_{2}}^{K}} \widetilde{J}_{K L, \Lambda_{2}, \Lambda_{3} ; \nu}^{\gamma_{C}} \hat{\tau}_{\Lambda_{3}, \Lambda_{4}}^{K I} .
\end{aligned}
$$

\section{COMPARISON TO THE COHERENT POTENTIAL APPROXIMATION CPA: ALLOYS WITH NO SHORT-RANGE ORDER}

We have implemented the formalism outlined above using the Munich self-consistent, spin polarized, relativistic KKR (SPRKKR) code of Ebert. ${ }^{53}$ Throughout we use an angular momentum cutoff $l_{\max }=3$ which is necessary for studies of transition metal systems with significant $d$-electron weight in the electronic structure close to the Fermi energy. The current matrix elements which occur in the conductivity expression have odd parity and couple for example $d$ states to both $p$ and $f$ states. Omission of the effect of the latter can lead to an underestimate of the conductivity. ${ }^{21,22}$ Although in principle the recently developed SCF-KKR-NLCPA method ${ }^{38}$ can provide the appropriate self-consistent one-electron charge densities and potentials, $\boldsymbol{\rho}_{\gamma_{C}}\left(\mathbf{r}_{I}\right)$ 's and $v_{\gamma_{C}}\left(\mathbf{r}_{I}\right)$, for our transport calculations, in these first applications, we use those generated by the faster, simpler SCF-KKR-CPA method $^{4,5}$ in order to explore the new aspects of our theory.

Owing to the deviation of its resistivity from the common Nordheim $[\rho \sim c(1-c)]$ behavior $^{54}$ and to earlier extensive studies made of it, ${ }^{16,22,55}$ the $\mathrm{AgPd}$ series of solid solutions provides the ideal initial test bed for our new method. We implement the formalism with NLCPA clusters containing four sites $\left(N_{C}=4\right)$ for this fcc based system. Figure 1 shows the calculations of the resistivities of randomly disordered, substitutional $\mathrm{Ag}_{c} \mathrm{Pd}_{1-c}$ alloys compared to those calculated 


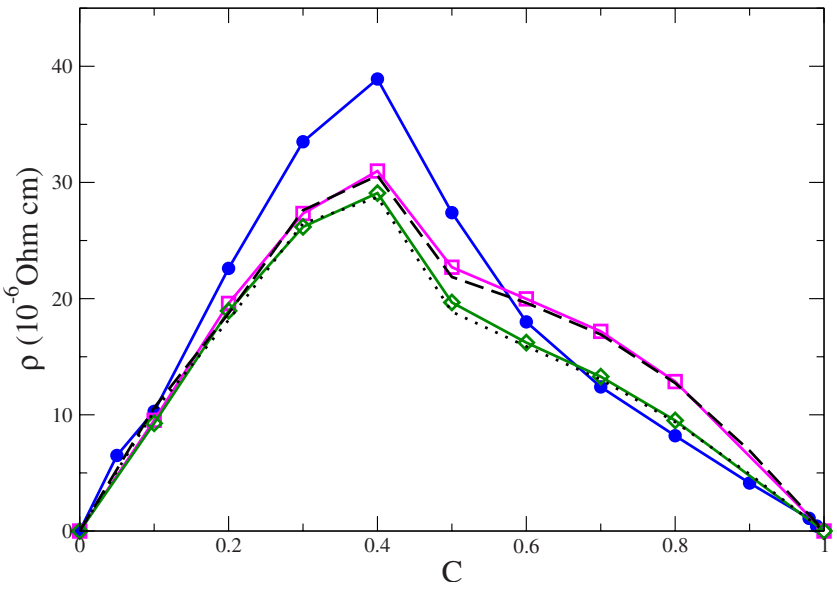

FIG. 1. (Color online) The resistivity of randomly disordered alloys $\operatorname{Ag}_{c} \mathrm{Pd}_{1-c}$ alloys as a function of concentration $c$. The full lines are the NLCPA results [pink (green) lines with squares (diamonds) show those without (with) vertex corrections]. The dashed curves show the CPA results [long dashes (dots)—without (with) vertex corrections]. The experimental results of Guenault (Ref. 58) are shown for comparison (full blue lines with filled circles).

using the established CPA formalism of Butler. ${ }^{21}$ Both implementations are presented with and without the so called "vertex corrections." We see that there is little difference between the two sets of calculations and the vertex corrections are found to be fairly insignificant for each approach. This aspect has been discussed fully in relation to the CPA calculations in earlier publications (e.g., (Refs. 56 and 57) and so will not be discussed further here. Both sets of calculations describe the experimental trends well and extensive work over two decades using the CPA formalism has shown how the trends of resistivities of randomly disordered transition metal alloys can be reliably described. The good agreement between our NLCPA results and those from the well established CPA method for these alloys where no short-range order is present is a very satisfactory first test of the new formalism. Figure 1 shows a pronounced asymmetry of both the experimental and theoretical curves. There is a shoulder emerging at $c$ $\approx 0.5$. As discussed, for example, by Stocks and Butler ${ }^{15}$ from a Boltzmann equation analysis, this feature is caused by the filling of the alloys' $d$ bands when enough $\mathrm{Ag}$ is added. For $c<0.5$, the Fermi surface of $\mathrm{Ag}_{c} \mathrm{Pd}_{1-c}$ is comprised of a $\Gamma$-centered sheet of high velocity electrons and a complicated flat $d$-band related structure near the Brillouin zone boundary. As $c$ approaches 0.5 , this structure shrinks and vanishes and there is an associated change in character from $d$ to $s p$ of the electrons associated with the major current carrying $\Gamma$-centered sheet.

Figure 1 also shows that the theoretical calculations underestimate the resistivity for the concentration region 0.2 $<c<0.55$. This is a typical feature of such calculations and we return later to a discussion of reasons for this following our study of the effects of SRO on the resistivity.

\section{EFFECTS OF SHORT-RANGE ORDER ON RESISTIVITY}

Many properties of alloys such as resistivity are affected by short-range order. Indeed, resistivity measurements are often used to monitor the changes in SRO which occur in annealing processes. If an alloy undergoes defect annealing after having been cold worked, there are significant changes in its physical properties owing to microstructural changes. For technical applications, it is important to know what these changes are so that physical properties can be controlled. SRO plays an important role in this and resistivity measurements are used to follow its kinetics. ${ }^{59}$ Our formalism is designed to help the interpretation of such measurements since it can describe the effects of short-range order on transport properties of alloys. It enables the calculation of the resistivity of a system to be made for a prescribed degree of SRO via the setting of the cluster configurational probabilities $P_{\gamma_{C}}$. Hence, it can aid the extraction of SRO attributes from resistivity measurements.

Our first application is to the bcc based series of disordered $\mathrm{Cu}_{c} \mathrm{Zn}_{1-c}$ alloys. We implement the NLCPA resistivity formalism using the smallest clusters and coarsest Brillouin zone tiling, i.e., $N_{C}=2$. This means correlations only between nearest neighbors can be described. We incorporate SRO according to the following three prescriptions for the four configurational weights, $P_{1}=P(\mathrm{CuCu}), \quad P_{2}=P(\mathrm{CuZn}), \quad P_{3}$ $=P(\mathrm{ZnCu})$, and $P_{4}=P(\mathrm{ZnZn})$ :

(a) No SRO, $P(\mathrm{CuCu})=c^{2}, P(\mathrm{CuZn})=P(\mathrm{ZnCu})=c(1-c)$, and $P(\mathrm{ZnZn})=(1-c)^{2}$.

(b) Short-range order (minimizing number of like nearest neighbors) for $c>0.5, \quad P(\mathrm{CuCu})=(2 c-1), \quad P(\mathrm{CuZn})$ $=P(\mathrm{ZnCu})=(1-c)$, and $P(\mathrm{ZnZn})=0$, and for $c<0.5$, $P(\mathrm{CuCu})=0, P(\mathrm{CuZn})=P(\mathrm{ZnCu})=c$, and $P(\mathrm{ZnZn})=(1-2 c)$.

(c) Short-range clustering (maximizing number of like nearest neighbors), $P(\mathrm{CuCu})=c, P(\mathrm{CuZn})=P(\mathrm{ZnCu})=0$, and $P(\mathrm{ZnZn})=(1-c)$.

Figure 2 summarizes our findings. Vertex corrections are included and are shown to be large for these systems. This concurs with earlier results for the randomly disordered alloys, which have low $d$-electron weight in electronic structure around the Fermi energy. In the absence of any shortrange ordering, the results show approximate adherence to the expected Nordheim $c(1-c)$ behavior and the results both with and without vertex corrections are very close to the CPA results and experimental results. ${ }^{60}$ Incorporating shortrange order with extent only between nearest neighbors decreases the resistivity as expected for all concentrations. The resistivity now follows a rough $c(1-c)+\lambda c^{2}(1-c)^{2}$ dependence so that the greatest reduction occurs at the stoichiometric concentration of $c=0.5$. Conversely, when shortranged clustering is included the resistivity increases for all concentrations and the $c(1-c)$ behavior returns. Evidently, current is enhanced on both types of atom when they are surrounded by unlike neighbors. These results are in good agreement with experimental measurements of the resistivity which show the resistivity to significantly decrease when the alloys are annealed so that short-ranged order is induced. ${ }^{60}$

We have also investigated the effect of short-range order on $\mathrm{Ag}_{c} \mathrm{Pd}_{1-c}$ alloys by once more choosing configurational weights such that the number of like neighbors is minimized for each concentration. Figure 3 contains the results. The effect of SRO is less than that found in CuZn. Below $c$ $=0.3$, the effect is negligible, whereas for larger concentrations, short-range order depresses the resistivity a little show- 


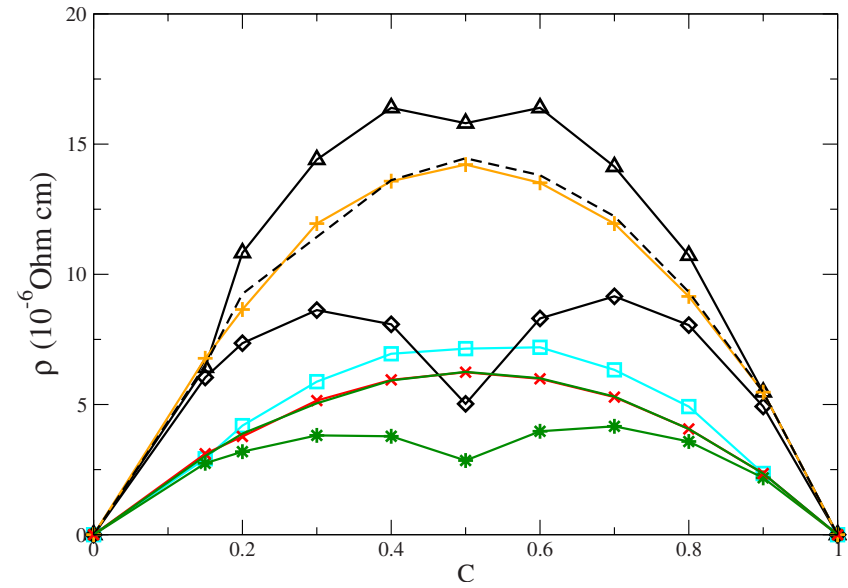

FIG. 2. (Color online) The resistivity of $\mathrm{Cu}_{c} \mathrm{Zn}_{1-c}$ alloys as a function of concentration $c$. The full (red) line with crosses shows the calculations when no SRO is included. [These are nearly indistinguishable from the CPA results (green line).] The (green) line with asterisks shows the NLCPA results when SRO is included and the square boxes (light blue line) show results when short-ranged clustering is included. Results are also shown where the vertex corrections have not been included: full (yellow) line with plus signs-NLCPA results for no SRO, (the dashed line shows the CPA results); lines with diamonds-NLCPA results including SRO; and lines with triangles-NLCPA results including short-ranged clustering.

ing how the current is enhanced on a site when surrounded by unlike neighbors. Experimental measurements on $\mathrm{Ag}_{c} \mathrm{Pd}_{1-c}$ alloys find that annealing has a smaller effect ${ }^{22,58}$ than in $\mathrm{Cu}_{c} \mathrm{Zn}_{1-c}$ in line with our calculations. It is also found that cold work causes little change to the resistivity suggest-

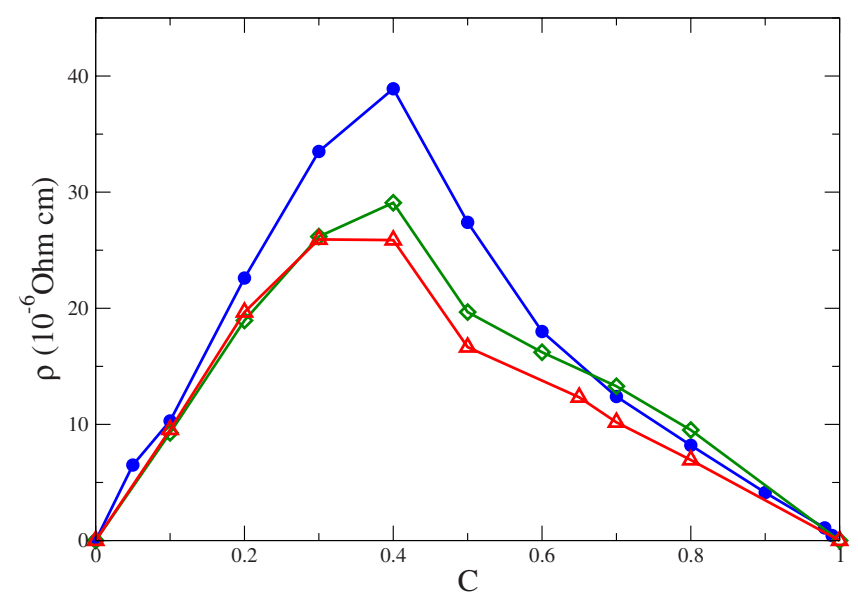

FIG. 3. (Color online) The resistivity of $\mathrm{Ag}_{c} \mathrm{Pd}_{1-c}$ alloys as a function of concentration $c$. The full (green) line with diamonds shows the calculations when no SRO is included. The (red) line with triangles shows those when SRO is included. Vertex corrections are included in both plots. The experimental data (Ref. 58) are also shown (blue line with filled circles). ing that additional defects such as dislocations may already be present affecting the measurements. This may be one reason of our underestimate of the resistivity, as shown in Figs. 1 and 3. Work is in progress to model this sort of effect. There are two chief remaining factors which may also contribute to the resistivity underestimate for $0.2<c<0.55$. The first concerns the effects on the resistivity of static displacements of the atoms from ideal fcc lattice positions. Since there is little size mismatch between $\mathrm{Ag}$ and $\mathrm{Pd}$ atoms, it is reasonable to estimate that this effect is small. The second factor is well documented ${ }^{61}$ and derives from the use of the adiabatic local density approximation (ALDA) in the time dependent density functional basis of our theory. It is possible therefore that the resistivity underestimate we find for strong scattering $\mathrm{Ag}_{c} \mathrm{Pd}_{1-c}$ alloys $(0.2<c<0.55)$, is partly caused by the ALDA not taking full account of disorderinduced enhancements of quasiparticle interactions.

\section{CONCLUSIONS}

Short-range ordering and clustering dramatically affect the transport properties of many alloys. Indeed resistivity measurements, which can be made easily and rapidly, provide a good way to monitor microstructural changes that occur in materials processing. In this paper, we have described a way to make quantitative calculations of the resistivity of disordered systems which possess short-range order or clustering. The $a b$ initio theory starts from the density functional theory for these systems recently devised by Rowlands et $a{ }^{38}{ }^{38}$ using the SCF-KKR-NLCPA electronic structure method. Our first calculations for $\mathrm{Cu}_{c} \mathrm{Zn}_{1-c}$ and $\mathrm{Ag}_{c} \mathrm{Pd}_{1-c}$ show the expected decrease of resistivity when short-range order is imposed, whereas short-ranged clustering produces an increase. For the randomly disordered alloys, the results are very similar to those that have been produced from established KKR-CPA resistivity calculations based on Butler et al. work. ${ }^{15,21}$ These first calculations have not included the effects of the short-range order on the selfconsistent charge densities and potentials that are available from the SCF-KKR-NLCPA method. So far, also short-range clustering and ordering effects over only the shortest nearest atomic neighbor range have been included. The computational development work is in progress to remove these current practical limitations. The counterintuitive behavior of the resistivities of the $K$-state alloys such as $\mathrm{NiCr}$, NiMo, and PdW will be ideal next systems to study.

\section{ACKNOWLEDGMENTS}

The authors are grateful to B. L. Györffy for very useful discussions. They also acknowledge financial assistance from the UK EPSRC and the SSP 1145 "MODERN AND UNIVERSAL FIRST-PRINCIPLES METHODS FOR MANY-ELECTRON SYSTEMS IN CHEMISTRY AND PHYSICS" as well as the SFB 689 "Spinphänomene in reduzierten Dimensionen" of the DFG. Computing facilities for this work were provided by the University of Warwick Centre for Scientific Computing and the Universität München. 
${ }^{1}$ P. Soven, Phys. Rev. 156, 809 (1967).

${ }^{2}$ P. Hohenberg and W. Kohn, Phys. Rev. 136, B864 (1964).

${ }^{3}$ W. Kohn and L. J. Sham, Phys. Rev. 140, A1133 (1965).

${ }^{4}$ D. D. Johnson, D. M. Nicholson, F. J. Pinski, B. L. Gyorffy, and G. M. Stocks, Phys. Rev. Lett. 56, 2088 (1986).

${ }^{5}$ D. D. Johnson, D. M. Nicholson, F. J. Pinski, B. L. Györffy, and G. M. Stocks, Phys. Rev. B 41, 9701 (1990).

${ }^{6}$ B. L. Györffy, Phys. Rev. B 5, 2382 (1972).

${ }^{7}$ G. M. Stocks, W. M. Temmerman, and B. L. Györffy, Phys. Rev. Lett. 41, 339 (1978).

${ }^{8}$ B. L. Györffy, D. D. Johnson, F. J. Pinski, D. M. Nicholson, and G. M. Stocks, in Proceedings of the NATO Advanced Study Institute on Alloy Phase Stability, edited by G. M. Stocks and A. Gonis (Kluwer, Dordrecht, 1987), p. 421.

${ }^{9}$ J. B. Staunton and B. L. Györffy, Phys. Rev. Lett. 69, 371 (1992).

${ }^{10}$ M. Lüders, A. Ernst, M. Däne, Z. Szotek, A. Svane, D. Ködderitzsch, W. Hergert, B. L. Györffy, and W. M. Temmerman, Phys. Rev. B 71, 205109 (2005).

${ }^{11}$ J. S. Faulkner, Prog. Mater. Sci. 27, 1 (1982).

${ }^{12}$ B. L. Györffy, B. Ginatempo, D. D. Johnson, D. M. Nicholson, F. J. Pinski, J. B. Staunton, and H. Winter, Philos. Trans. R. Soc. London, Ser. A 334, 515 (1991).

${ }^{13}$ I. D. Hughes et al., Nature (London) 446, 650 (2007).

${ }^{14}$ J. B. Staunton, S. Ostanin, S. S. A. Razee, B. L. Gyorffy, L. Szunyogh, B. Ginatempo, and E. Bruno, Phys. Rev. Lett. 93, 257204 (2004).

${ }^{15}$ G. M. Stocks and W. H. Butler, Phys. Rev. Lett. 48, 55 (1982).

${ }^{16}$ W. H. Butler and G. M. Stocks, Phys. Rev. B 29, 4217 (1984).

${ }^{17}$ W. H. Butler, Phys. Rev. B 29, 4224 (1984).

${ }^{18}$ B. Velický, Phys. Rev. 184, 614 (1969).

${ }^{19}$ R. Kubo, J. Phys. Soc. Jpn. 12, 570 (1957).

${ }^{20}$ D. A. Greenwood, Proc. Phys. Soc. London 71, 585 (1958).

${ }^{21}$ W. H. Butler, Phys. Rev. B 31, 3260 (1985).

${ }^{22}$ J. C. Swihart, W. H. Butler, G. M. Stocks, D. M. Nicholson, and R. C. Ward, Phys. Rev. Lett. 57, 1181 (1986).

${ }^{23}$ J. Banhart, R. Bernstein, J. Voitländer, and P. Weinberger, Solid State Commun. 77, 107 (1991).

${ }^{24}$ J. Banhart, H. Ebert, P. Weinberger, and J. Voitländer, Phys. Rev. B 50, 2104 (1994).

${ }^{25}$ A. Gonis, Green Functions for Ordered and Disordered Systems, Studies in Mathematical Physics Vol. 4 (North-Holland, Amsterdam, 1992).

${ }^{26}$ H. Thomas, Z. Phys. 129, 219 (1951).

${ }^{27}$ A. Mookerjee and R. Prasad, Phys. Rev. B 48, 17724 (1993).

${ }^{28}$ T. Saha, I. Dasgupta, and A. Mookerjee, Phys. Rev. B 50, 13267 (1994).

${ }^{29}$ T. Saha, I. Dasgupta, and A. Mookerjee, J. Phys.: Condens. Matter 8, 1979 (1996).

${ }^{30}$ J. Kudrnovsky and V. Drchal, Phys. Rev. B 41, 7515 (1990).

${ }^{31}$ A. Mookerjee, J. Phys. C 6, 1340 (1973).

${ }^{32}$ L. J. Kaplan and T. Gray, Phys. Rev. B 14, 3462 (1976).

${ }^{33}$ R. Haydock, V. Heine, and M. Kelly, J. Phys. C 5, 2845 (1972).

${ }^{34}$ K. K. Saha, A. Mookerjee, and O. Jepsen, Phys. Rev. B 71,
094207 (2005).

${ }^{35}$ K. Tarafder, A. Chakrabarti, K. K. Saha, and A. Mookerjee, Phys. Rev. B 74, 144204 (2006).

${ }^{36}$ D. A. Rowlands, J. B. Staunton, and B. L. Györffy, Phys. Rev. B 67, 115109 (2003).

${ }^{37}$ D. A. Rowlands, J. B. Staunton, B. L. Györffy, E. Bruno, and B. Ginatempo, Phys. Rev. B 72, 045101 (2005).

${ }^{38}$ D. A. Rowlands, A. Ernst, B. L. Györffy, and J. B. Staunton, Phys. Rev. B 73, 165122 (2006).

${ }^{39}$ D. A. Biava, S. Ghosh, D. D. Johnson, W. A. Shelton, and A. V. Smirnov, Phys. Rev. B 72, 113105 (2005).

${ }^{40}$ S. Ghosh, D. A. Biava, W. A. Shelton, and D. D. Johnson, Phys. Rev. B 73, 085106 (2006).

${ }^{41}$ D. Ködderitzsch, H. Ebert, D. A. Rowlands, and A. Ernst, New J. Phys. 9, 81 (2007).

${ }^{42}$ M. Jarrell and H. R. Krishnamurthy, Phys. Rev. B 63, 125102 (2001).

${ }^{43}$ M. H. Hettler, A. N. Tahvildar-Zadeh, M. Jarrell, T. Pruschke, and H. R. Krishnamurthy, Phys. Rev. B 58, R7475 (1998).

${ }^{44}$ M. H. Hettler, M. Mukherjee, M. Jarrell, and H. R. Krishnamurthy, Phys. Rev. B 61, 12739 (2000).

${ }^{45}$ Th. Maier, M. Jarrell, Th. Pruschke, and J. Keller, Eur. Phys. J. B 13, 613 (2000).

${ }^{46}$ M. Tsukada, J. Phys. Soc. Jpn. 32, 1475 (1972).

${ }^{47}$ P. R. Tulip, J. B. Staunton, D. A. Rowlands, B. L. Györffy, E. Bruno, and B. Ginatempo, Phys. Rev. B 73, 205109 (2006).

${ }^{48}$ G. M. Batt and D. A. Rowlands, J. Phys.: Condens. Matter 18, 11031 (2006).

${ }^{49}$ E. Runge and E. K. U. Gross, Phys. Rev. Lett. 52, 997 (1984); E. K. U. Gross and W. Kohn, ibid. 55, 2850 (1985); E. K. U. Gross et al., in Density Functional Theory, Springer Series Topics in Current Chemistry, edited by R. F. Nalewajski (Springer, Berlin, 1996).

${ }^{50}$ W. G. Henry and P. A. Schroeder, Can. J. Phys. 41, 1076 (1963).

${ }^{51}$ J. S. Faulkner and G. M. Stocks, Phys. Rev. B 21, 3222 (1980).

${ }^{52}$ M. Hwang, A. Gonis, and A. J. Freeman, Phys. Rev. B 35, 8985 (1987).

${ }^{53}$ H. Ebert, in Electronic Structure and Physical Properties of Solids, Lecture Notes in Physics Vol. 535, edited by H. Dreysse (Springer, Berlin, 2000) and references therein; The Munich SPRKKR band structure program package, 2005 (http:// olymp.cup.uni-muenchen.de/ak/ebert/SPRKKR).

${ }^{54}$ L. Nordheim, Ann. Phys. 9, 664 (1931).

${ }^{55}$ J. Banhart and H. Ebert, Solid State Commun. 94, 445 (1995).

${ }^{56}$ I. Turek, J. Kudrnovsky, V. Drchal, and P. Weinberger, J. Phys.: Condens. Matter 16, 5607 (2004).

${ }^{57}$ J. Banhart, Philos. Mag. B 77, 105 (1998).

${ }^{58}$ A. M. Guenhault, Philos. Mag. 30, 641 (1974).

${ }^{59}$ M. Migschitz, W. Garlipp, and W. Pfeiler, Acta Mater. 44, 2831 (1996).

${ }^{60}$ C. Y. Ho, J. Phys. Chem. Ref. Data 12, 183 (1983).

${ }^{61}$ R. H. Brown, P. B. Allen, D. M. Nicholson, and W. H. Butler, Phys. Rev. Lett. 62, 661 (1989). 